\title{
Overall Monitoring and Diagnosis for Hybrid Vehicle Powertrains
}

\author{
Christofer Sundström*, Erik Frisk*, and Lars Nielsen* \\ * Vehicular Systems, Dept. of Electrical Engineering, Linköping \\ University, SE-58183 Linköping, Sweden, \{csu,frisk,lars\}@isy.liu.se.
}

\begin{abstract}
Designing diagnosis systems for hybrid vehicles include new features compared to conventional vehicles, e.g. mode switches in the system. The influence of this on the performance of the diagnosis system is investigated by design and implementation of diagnosis systems on vehicle level. The diagnosis systems are based on two sensor configurations, one consisting of many sensors and one of few sensors. The diagnosis systems detect specific faults, here specifically faults in the electrical components in a hybrid vehicle driveline, but the methodology is generic. There is a connection between the design of the energy management and the diagnosis system, and this interplay is of special relevance when models of components are valid only in some operating modes. In the systems implemented, the diagnosis system based on few sensors is more complex and includes a larger part of the vehicle model than the system based on more sensors.
\end{abstract}

Keywords: sensor placement; sensor configuration; dynamic residual generator; parallel hybrid

\section{INTRODUCTION}

There are possibilities to increase the efficiency of automotive drivelines using hybrid technology. The highest relative fuel saving can be obtained in city buses and garbage trucks with many start and stops, but also a small relative saving in the fuel consumption for long haulage trucks results in a large amount of fuel. When hybridizing a vehicle, new components are added compared to a conventional vehicle, e.g. electric machines, battery, and power electronics. These components need to be monitored with the same accuracy as the components used in a conventional vehicle.

One reason for monitoring the system is safety. Faults in the electrical system may be fatal due to the high voltage in the system. It is also possible that a truck starts to move from stand still, due to a fault in the system, resulting in that a torque is applied by the electric machine. It is also of relevance to protect components from breaking down if a fault occurs, and here it is especially important to protect the battery due to its high cost.

The demands on the diagnosis systems in a conventional vehicle has been increased over a long period of time. Therefore such diagnosis systems have been developed and refined step by step to achieve the performance of today's systems. In a hybrid electric vehicle (HEV), many new components are added. Monitoring these leads to new challenges since there are many different operating modes in an HEV, and there is a freedom in choosing operating points of the components via the energy management. One example is that the electric components are either active or not.

The objective of this work is to study key topics for overall monitoring and diagnosis on vehicle level of hybrid vehicles. A main topic is a study on how the choice of the sensor configuration affects the model based diagnosis system.

\section{SIMULATION PLATFORM}

To investigate the interplay between vehicle, control, and driver with emphasis on monitoring and diagnosis, a simulation platform in Simulink has been developed. The simulation platform includes descriptions of the truck, driver model, control and energy management algorithms, and different diagnosis systems. The diagnosis framework used in the paper is consistency based diagnosis using precompiled tests, or residuals, see for example Blanke et al. (2006) or the references therein. For logical foundations of the approach, see for example de Kleer et al. (1992).

The structure of the platform is given in Figure 1. The vehicle model is based on models of the components with a fixed interface to be able to easily change a component model without modifying the rest of the model. In Figure 1, the lower dashed line has a special meaning in that the levels above are simulated as a time-continuous system, whereas the level below is simulated at a fix sample rate. Here, the diagnosis system is updated at a rate of $80 \mathrm{~Hz}$, since this reflects a real application. In the platform, the models of the vehicle, environment and driver are based on the model library developed in the Center for Automotive Propulsion Simulation (CAPSim, 2009). Modifications to these models are carried out to model a truck instead of a passenger car, and to include the possibility to induce faults in the models and to add sensor noise.

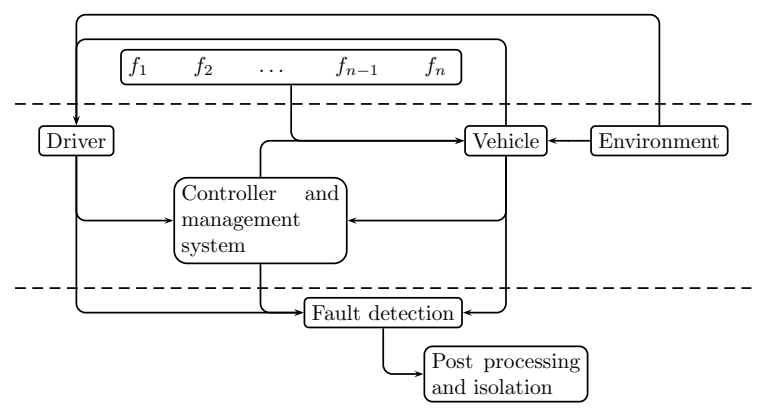

Fig. 1. The structure of the implemented platform. Above the first horizontal line the faults are modeled, between the lines the models of the vehicle, driver, and environment, and the lowest level contains the diagnosis system described in Sections 3 and 4 . 


\subsection{Environment}

The environment contains information about the driving cycle, and ambient temperature and pressure. In the simulations the driving cycle FTP75 is used.

\subsection{Driver model}

The model of the driver is a PI-controller using the information from the actual speed and the reference speed from the driving cycle. The model also handles gear selection and when to engage or disengage the clutch.

\subsection{Vehicle model}

The vehicle modeled is a long haulage truck with a total weight of $40.000 \mathrm{~kg}$. The configuration of the driveline is a parallel hybrid, see Figure 2.

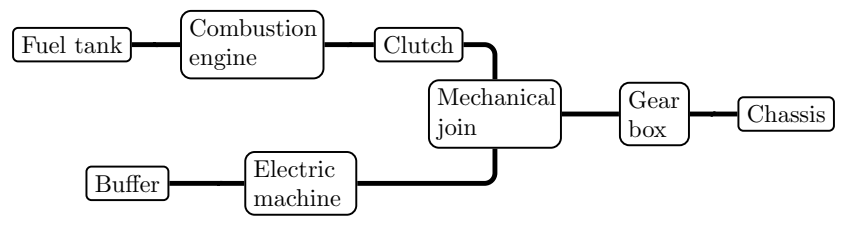

Fig. 2. The modeled truck is a parallel hybrid with the connection of the electrical and conventional parts of the driveline between the clutch and the gearbox.

Since the objective with this work is to study the interaction between the components in the vehicle, it is preferable to use basic component models. It is though easy to add more advanced models of the components. The models of the battery, electric machine, power electronics, and the clutch are described below, since these components are of interest in the diagnosis systems. The other components are described in Sundström et al. (2010).

Battery The battery is modeled as a voltage source and an inner resistance. The capacity and nominal voltage are $9 \mathrm{kWh}$ and $256 \mathrm{~V}$, respectively.

Electric machine The electric machine is able to convert electric power to mechanical power and vice verse. A voltage, $U_{e m}$, is applied on the component, resulting in a torque on the outgoing shaft. The machine used is the one in CAPSim, where the model is that the torque $T_{e m}$ is proportional to the armature current, $I_{e m}$, and that the speed of the machine, $\omega_{e m}$, strongly is connected to the voltage $U_{e m}$

$$
\begin{aligned}
I_{e m} & =\frac{U_{e m}-k_{a} \omega_{e m}}{R} \\
T_{e m} & =I_{e m} k_{i}
\end{aligned}
$$

The model is parametrized as a $33 \mathrm{~kW}$ DC machine with constant magnetic flux with parameter values of the resistance, $R$, torque constant, $k_{i}$, and speed constant, $k_{a}$, are set to $0.044 \Omega, 0.50 \mathrm{Nm} / \mathrm{A}$, and $0.51 \mathrm{Vs} / \mathrm{rad}$, respectively. In an ideal machine, $k_{i}$ and $k_{a}$ are equal, and are defined by $K \Phi$, where $K$ is a machine constant that depends on the design parameters of the machine, and $\Phi$ is the magnetic flux produced by the stator. In HEVs it is common to use a permanent magnet synchronous machine (Chau et al., 2008) due to its high efficiency, and models according to Guzzella and Sciarretta (2007) and others are to be included in the platform.
Power electronics The model of the power electronics is included in the electric machine and is assumed to be an ideal component

$$
P_{b}=P_{e m} \Longleftrightarrow I_{b} U_{b}=I_{e m} U_{e m}
$$

where $P_{b}$ and $P_{e m}$ are the electrical power from the battery and motor, and $U_{b}$ and $I_{b}$ the voltage and current in the battery.

Clutch There is a model of the clutch to handle starts and gear shifts. When the clutch is engaged or disengaged, the clutch is modeled as an ideal component, but the model is more complex when there is a difference in the rotational speeds on the in- and outgoing axles.

\subsection{Controller and energy management}

There are several approaches to develop a well performing energy management, e.g. the global optimal solution (Lin et al., 2003) using dynamic programming, or finding equivalent-consumption minimization strategies (ECMS) (Sciarretta and Guzzella, 2007). In this investigation a less complex energy management is used in order to focus on the diagnosis systems.

The basic idea in the developed energy management is to charge the battery via the electric machine instead of using the mechanical brakes and later use this energy as a complement to the combustion engine. To be able to store as much energy as possible during a retardation, it is preferred to have a low state of charge, $S o C$, in the battery before the retardation. This is achieved by primarily propel the vehicle via the electrical machine if $S o C$ is larger than a predefined threshold, $S o C_{l o w}$.

\subsection{Sensors}

The truck is assumed to be equipped with sensors measuring voltages, currents and rotational velocities. In addition to these sensors, the torque from the electric machine is measured in one of the diagnosis systems. This is to investigate the influence of using many sensors measuring signals of the electrical components.

\subsection{Measurement noise}

Noise is added to all sensor signals with arbitrary amplitude. To simulate measurement noise, a Band-Limited White Noise block that is updated at $80 \mathrm{~Hz}$ is used in the Simulink model.

\subsection{Faults}

To model that the battery, power electronics, or the electric machine may break down, two parameter values and two voltages in these models have the possibility to be modified. Note that these faults are only examples of how a fault in these components can be represented in the model. The following modifications of the signals are introduced to model the faults where the nominal signals are denoted by the superscript nom:

$$
\begin{aligned}
f_{\text {em }, k i}: & k_{i}=\left(1+f_{\text {em }, k i}\right) k_{i}^{n o m} \\
f_{\text {em }, R}: & R=\left(1+f_{\text {em }, R}\right) R^{\text {nom }} \\
f_{p e}: & U_{\text {em }, \text { control }}=\left(1+f_{\text {pe }}\right) U_{\text {em }, \text { control }}^{\text {nom }} \\
f_{b, s c}: & U_{b}=\left(1+f_{b, s c}\right) U_{b}^{\text {nom }}
\end{aligned}
$$

where $f_{b, s c}$ models that not all cells in the battery are used due to an internal short circuit, $f_{p e}$ is a fault in the power electronics, and $f_{e m, k i}$ and $f_{e m, R}$ are two faults in the electric machine. 
Sensor faults are modeled as an offset fault, e.g. for the voltage sensor

$$
U_{\text {em,sens }}=U_{\text {em }}+f_{\text {em }, \text {,sens }}
$$

where $f_{\text {em, } U \text {,sens }}$ possibly is time variant.

When a fault is induced in the model, the value of the fault is given in Table 1.

Table 1. Values of faults induced in the model.

The voltage $U_{e m}$ varies in the range $0-200 \mathrm{~V}$, $U_{b} \approx 250 \mathrm{~V}$ and $\omega_{g b}<50 \mathrm{rad} / \mathrm{s}$.

\begin{tabular}{rl} 
Faults & Value \\
\hline$f_{\text {em }, k i}$ & -0.5 \\
$f_{\text {em, }, R}$ & -0.5 \\
$f_{p e}$ & -0.5 \\
$f_{b, s c}$ & -0.5 \\
$f_{b, U, s e n s}$ & $20 \mathrm{~V}$ \\
$f_{\text {em,U,sens }}$ & $20 \mathrm{~V}$ \\
$f_{\omega, g b}$ & $20 \mathrm{rad} / \mathrm{s}$ \\
\hline
\end{tabular}

\section{SENSOR CONFIGURATIONS AND THEORETICAL MAXIMUM FAULT ISOLABILITY}

To analyze how the choice of sensor configuration affects the performance and complexity of the diagnosis system, two systems using different sensor configurations are developed. One set of sensors is chosen to achieve a diagnosis system that is easy to design, and the other set is chosen to use as few sensors as possible. The sensor configurations will also be investigated to see how the sensor noise affects the performance of the diagnosis.

\subsection{Sensor configuration 1}

The first system includes sensors that measure signals close to the components that are to be monitored, i.e. the battery, electric machine and power electronics:

- $T_{\text {em,sens }}$ - torque from electric machine

- $\omega_{g b, s e n s}$ - outgoing speed of gear box

- $I_{b, \text { sens }}$ - current to battery

- $I_{\text {em,sens }}$ - current in electric machine

- $U_{\text {em,sens }}$ - voltage of electric machine

Note that in this system a torque sensor in the electric machine is used. Torque sensors are normally not used in series production, but in this case the torque sensor is included in the system to investigate its impact on the performance of the diagnosis.

Given a model and a set of sensors it is possible to determine what detectability and isolability of the faults that are theoretically possible to achieve. In Krysander and Frisk (2008) this is done by a structural analysis (Dustegör et al., 2006; Blanke et al., 2006) of the model. The method is based on that all variables that are used in every equation are listed. How the variables are included (e.g. linear, exponential, differentiated) is not of importance in this analysis. The structural model using the above described sensor configuration is shown in Figure 3, where the last five equations represent the sensor equations and are modified if a different sensor configuration is used. Given the set of sensors described above it is possible, under the assumptions in Krysander and Frisk (2008), to achieve full isolability of the faults described in Section 2.7.

\subsection{Sensor configuration 2}

In the second set of sensors used, the number of sensors to achieve full isolability is minimized to be able to analyze

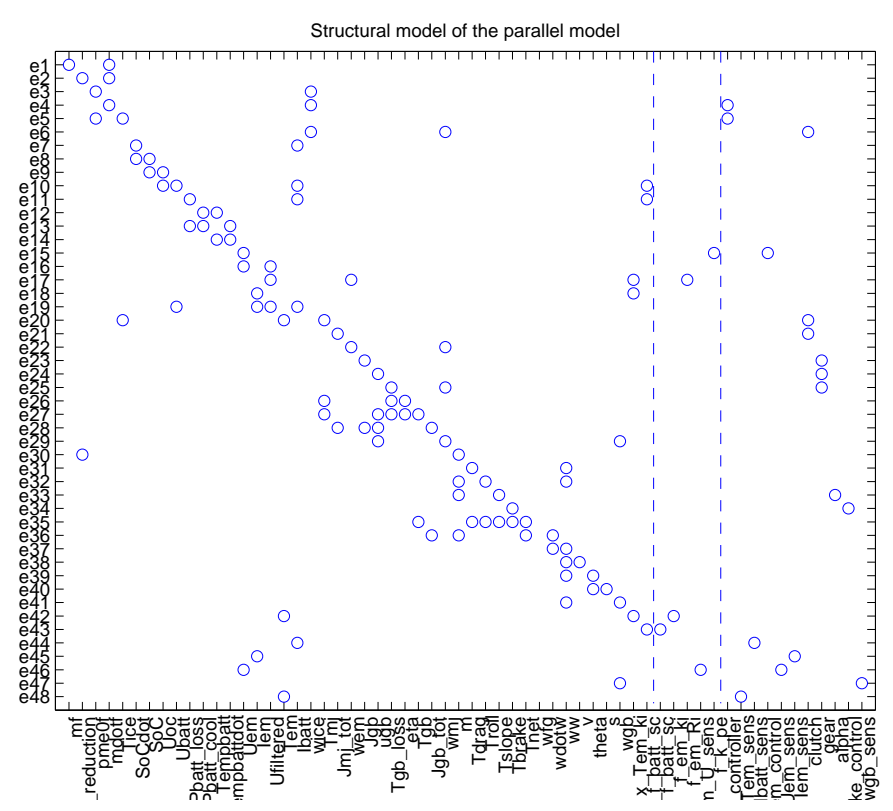

Fig. 3. The structural model of the truck where 5 sensors are used in the model. Each row represents an equation, each column a variable, and the circles indicates which variables that are included in each equation. The variables to the left of the dashed lines are unknowns, between the lines are possible faults, and to the right the known variables such as sensor signals and signals from the controllers.

the impact this choice has on the performance of the diagnosis system. To find this set, a sensor placement algorithm (Krysander and Frisk, 2008) is run using the structural model. If all sensors used are to be monitored, in addition to the faults described in Section 2.7, three sensors are required to isolate these faults. There are several different sets with three sensors that fulfills this, and in the diagnosis system the following sensor configuration is used:

- $\omega_{g b, s e n s}$ - outgoing speed of gearbox

- $U_{b, s e n s, a}$ - voltage of battery

- $U_{b, \text { sens }, b}$ - voltage of battery

As seen there are two sensors measuring the voltage over the battery. This is required to be able to isolate faults in the voltage sensors.

\section{DIAGNOSIS SYSTEMS DESIGN}

Two model based diagnosis systems, using the sensor sets described above, are implemented in the platform (Figure 1), monitoring the faults in Section 2.7 without a model describing how the faults affect the variable. This means that how the faults affect the signals in the vehicle model is not known in the diagnosis systems, which is an advantage since the tests react to any variation to the fault free model.

A diagnosis system is based on one or several test that consists of a subset of the equations used in the vehicle model. There are many such overdetermined sets of equations, that are sensitive to different sets of faults. The minimal sets of equations that form an overdetermined part can be found using the structural model. Combinations of overdetermined parts of the model are proposed by an algorithm to achieve maximum isolability of the system in combination while using as few tests as possible. 


\subsection{Diagnosis system 1}

In the first diagnosis system, five sensors are used as described in Section 3.1. There are several combinations of tests that require four tests to achieve full isolability, and one of these combinations is chosen as the basis to diagnosis system 1 . The tests in this diagnosis system are relatively small (see Table 2), 2-14 equations are used in each test. The sets of equations that are used in the tests form substitution chains, i.e. there are no algebraic loops that need to be solved. This leads to that it is easier to design the residual generators.

As an example the first test in the diagnosis system is discussed. It is based on 2 equations and includes a sensor equation

$$
r=U_{\text {em,sens }}-U_{\text {em }}
$$

and the assumption that the power electronics works properly

$$
U_{\text {em }}=U_{\text {em, control }}
$$

This test is sensitive for $f_{p e}$ and $f_{e m, U, s e n s}$. The information about which tests that are expected to react on each fault is summarized in a decision structure in Table 3 . Test 1 reacts for example on $f_{p e}$ and $f_{e m, U, s e n s}$, which also can be seen in (4) and (5).

Table 2. Number of equations in the tests in the two diagnosis systems.

\begin{tabular}{l|ccl} 
& \# tests & \# sensors & \# equations \\
\hline System 1 & 4 & 5 & $2,9,14,12$ \\
System 2 & 6 & 3 & $2,15,29,35,35,36$ \\
\hline
\end{tabular}

Table 3. Decision structure in diagnosis system 1 .

\begin{tabular}{c|ccccc} 
& $f_{e m, k i}$ & $f_{e m, R}$ & $f_{p e}$ & $f_{b, s c}$ & $f_{\text {em }, U, s e n s}$ \\
\hline$T 1$ & & & $\mathrm{X}$ & & $\mathrm{X}$ \\
$T 2$ & $\mathrm{X}$ & $\mathrm{X}$ & $\mathrm{X}$ & $\mathrm{X}$ & \\
$T 3$ & & $\mathrm{X}$ & & $\mathrm{X}$ & $\mathrm{X}$ \\
$T 4$ & $\mathrm{X}$ & & & \\
\hline
\end{tabular}

If Test 1 alarms this is explained by either $f_{p e}$ or $f_{\text {em, } U \text {,sens }}$. If also Test 2 alarms the only possible explanation for this is that the power electronics is broken, given that the only possibility is single faults. Full fault isolation is possible since each fault influences different sets of tests (Blanke et al., 2006).

Model not valid In the second test, $\omega_{e m}$ is estimated via the angular speed sensor at the outgoing shaft of the gearbox, $\omega_{g b \text {,sens }}$ and the gear ratio, $u_{g b}$, according to

$$
\omega_{e m}=u_{g b} \omega_{g b, s e n s}
$$

The noise in $\omega_{g b \text {,sens }}$ is amplified with the gear ratio when $\omega_{e m}$ is estimated that later is used in the residual generator. To get equal test significance for all gears, varying noise levels has to be considered. Here a simple approach is adopted where the test is not valid for gears 1-4 where $u_{g b}$ is large.

CUSUM Due to sensor noise, the residuals will be nonzero even in a fault free case. To handle this a standard algorithm called CUSUM (Page, 1954) is used. The algorithm is based on that a signal, $s$, is constructed that has a negative expectation value in a fault free case and positive when a fault has occurred. The trend of a cumulative sum, $g$, of $s$ will then contain information about the status of the monitored system. The test quantity, $T$, is calculated as

$$
\begin{aligned}
s(t) & =|r(t)|-\nu \\
g(t+1) & =g(t)+s(t) \\
T(t) & =g(t)-\min _{0 \leq i<t} g(i)
\end{aligned}
$$

where $\nu$ is an offset that ensures that $E\{s(t)\}<0$ in the fault free case. The size of $\nu$ reflects the model uncertainty and noise in the model. The system alarms if $T>J$, where $J$ is a threshold and a design parameter, that is set to avoid false alarms and still achieve fast fault detection.

\subsection{Diagnosis system 2}

The second system uses as few sensors as possible that still achieve full isolability in the system according to the structural analysis. This choice is made to investigate the impact regarding performance of the system, but also the complexity in designing the system. All three sensors are monitored, resulting in that there are 7 fault modes monitored in diagnosis system 2 .

To achieve full isolability 6 tests are needed in this diagnosis system compared to 4 in system 1 . To investigate the influence of component models, the tests are not valid in all operating modes. For example, the model of the clutch used in the residual generators is only valid when the clutch is engaged. The models of the clutch and combustion engine are included in 4 of the tests in system 2 (see Figure 2 for the vehicle configuration). These 4 tests include a derivative of a signal, which could lead to problems since the signal is noisy.

The tests in this system include in general more equations than the tests in system 1 . Up to 36 equations are used in the tests (see Table 2). Note that the entire vehicle model consists of 46 equations. The decision structure for the system is given in Table 4 .

Algebraic loops In 5 of the 6 sets of equations, substitution chains form the residual generators. In Test 4 an algebraic loop has to be solved since the following two equations are included in the test

$$
\begin{aligned}
I_{e m} & =\frac{U_{e m}-\omega_{e m} k_{a}}{R} \\
U_{e m} & =\frac{I_{b} U_{b}}{I_{e m}}
\end{aligned}
$$

with the solution

$$
I_{e m}=-\frac{\omega_{e m} k_{a}}{2 R} \pm \sqrt{\left(\frac{\omega_{e m} k_{a}}{2 R}\right)^{2}+\frac{I_{b} U_{b}}{R}}
$$

These equations are only a small part of the residual generator, and the entire consistency relation is given in Sundström et al. (2010).

There are two possible solutions to $I_{e m}$, and both solutions are valid in different operating modes. In this case, the consistency based solution is to alarm when none of the solutions are consistent with measurement data. Thus, two residuals are calculated and the residual with the lowest magnitude is used in CUSUM to calculate the test quantity. It may happen that a fault that the test should react on has occurred and the residual corresponding to the incorrect solution of $I_{e m}$ is small. In such a situation, even though the residual for the correct $I_{e m}$ solution reacts, it may take longer for the CUSUM algorithm to accumulate and react. This is a typical drawback when using a sensor configuration with few sensors, since larger parts of the model are used increasing the possibility of multiple solutions.

Dynamic residual generators Four of the six tests include dynamics in the consistency relation. This means that a 
sensor signal, or a signal estimated from control signals and other sensors, appears in differentiated form. To avoid problems from differentiating noise, a transformation is made since the residual can be expressed in the form $r=a \dot{\omega}_{g b}+b$, where $a$ is a constant and $b$ is an arbitrary function of known signals (Frisk and Nyberg, 2001). The transformation includes a low pass filter of the residual generator which is expressed as:

$$
r=\frac{\alpha}{p+\alpha}\left(a \dot{\omega}_{g b}+b\right)
$$

using the state

$$
\omega=r-\alpha a \omega_{g b}
$$

we obtain

$$
\begin{aligned}
\dot{\omega} & =\alpha\left(r-\alpha a \omega_{g b}\right)+\alpha b \\
r & =\omega+\alpha a \omega_{g b}
\end{aligned}
$$

Model not valid The model of the clutch in the diagnosis system is only valid when the clutch is engaged, and in this operating mode, the model is that the torques and speeds on both sides of the clutch are equal. This results in that if the clutch is disengaged or there is a slip in the clutch, the four tests that include the model of the clutch are not valid. After the clutch pedal is released by the driver, the residuals in the corresponding tests are not updated in 3 seconds.

Tests 5 and 6 are noise sensitive for small $U_{e m}$, so voltages close to zero needs to be handled. Therefore the tests are not updated when $\left|U_{e m}\right|<1 \mathrm{~V}$ respectively $10 \mathrm{~V}$ depending on the test.

Reinitialization of the state in the low pass filter If a test has been "not valid" due to one of the reasons described above, the test has to be reactivated and the state in the residual generator (12) has to be reinitialized. When the state is reinitialized, it is assumed that the system is fault free and $r=0$ in (11). This leads to an error in the state if the system is faulty since then the assumption that $r=0$ is incorrect. The signal $\omega_{g b}$ is noisy and therefore an error in the initialization of the state may occur even in a fault free case. To decrease this error, the $\omega_{g b}$ that is used in the initialization of the state is filtered. The error will fade out with time and the residual will be non-zero after some time if the system is faulty. During the fade out period, the CUSUM algorithm is not updated during 10 seconds after the test has become valid, in order to avoid an erroneous decision whether the test is to react or not. The drawback with this is that the tests that use a dynamic residual generator and filter may be inactive a significant part of the time, thereby reducing the performance of the system. For example, a test that includes all limitations described above is only updated during $30 \%$ of the simulated test cycle.

Table 4. Decision structure in diagnosis system 2 .

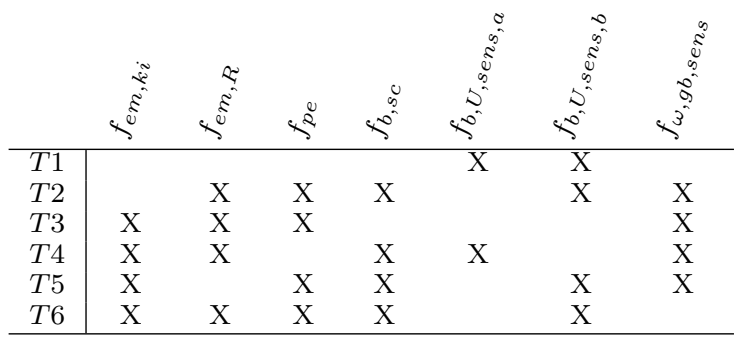

\section{RESULTS AND DISCUSSION}

To evaluate the performance of the diagnosis systems, simulations of a long haulage truck are carried out. The type of problems that are handled here, e.g. the impact of the number of sensors on the performance of the diagnosis, and the interplay between diagnosis and the energy management, are of interest, since these issues will also occur in reality when developing diagnosis systems. In the simulations, the faults are induced one by one to evaluate performance.

The performance, of the diagnosis systems vary, e.g. time to detect and isolate a fault. The system based on few sensors is e.g. more dependent on the energy management compared to the other system.

\subsection{Diagnosis system 1}

The diagnosis system based on 5 sensors has 5 states, where 2 states are used for iterative equation solving. In addition there are 4 states, one for each test, for the CUSUM tests. Normalized test quantities, $T_{\text {norm }}$, are calculated using the parameters in CUSUM (Section 4.1)

$$
T_{\text {norm }}=\frac{T}{J}
$$

and the test alarms if $T_{\text {norm }}>1$. The system performs as expected, and all faults are detected and isolated in a few seconds.

As an illustration, Figure 4 shows $T_{\text {norm }}$ when $f_{e m, R}$ is induced in the model after 400 seconds. Tests 2 and 3 react on this fault, as expected according to the decision structure in Table 3. The performance of the system detecting $f_{e m, R}$, is representative for all faults that are to be detected.

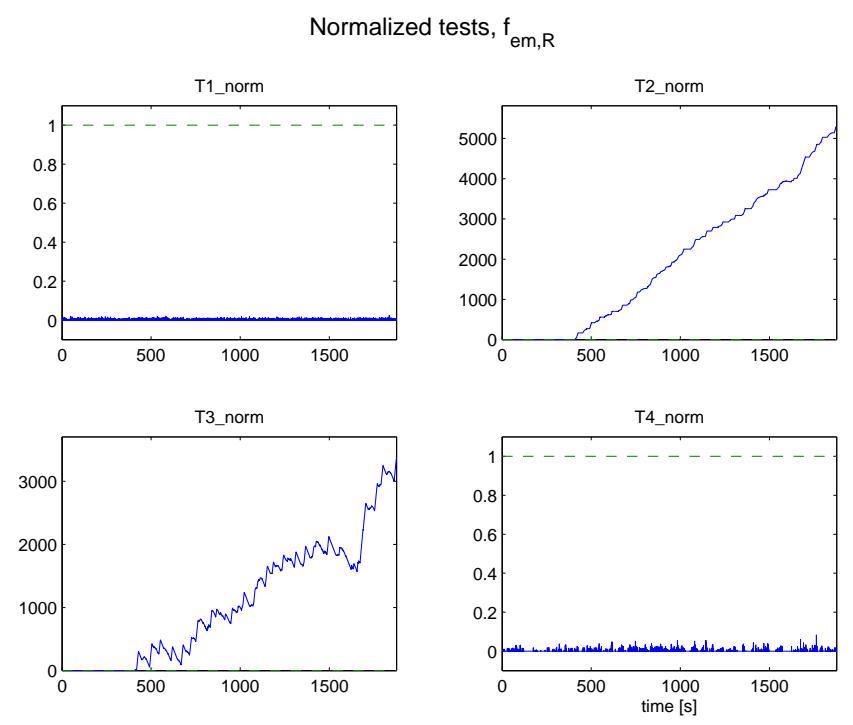

Fig. 4. The tests alarm if the normalized test quantity is larger than one. The fault $f_{e m, R}$ is induced in the model after 400 seconds and Test 2 and 3 react as expected.

\subsection{Diagnosis System 2}

Diagnosis system 2 is based on 3 sensors and has 33 states, where 10 states are used for iterative equation solving. In addition there are 6 states, one for each test, for the CUSUM tests. Normalized test quantities are calculated as in (14). 
All faults are detected. However, only five of the faults are fully isolated. The reasons are as follows. When the torque constant in the electric machine has changed, i.e. the fault $f_{e m, k i}$, this is not detected in Test 6 as expected from the structural analysis and the decision structure in Table 4. This means that this fault can not be isolated from $f_{\omega, g b, s e n s}$. Also when the resistance, i.e. $f_{e m, R}$ also in the electric machine, has changed is not detected in Test 4 see Figure 5. This means that this fault can not be isolated from $f_{p e}$. Improvements can be sought by using variable
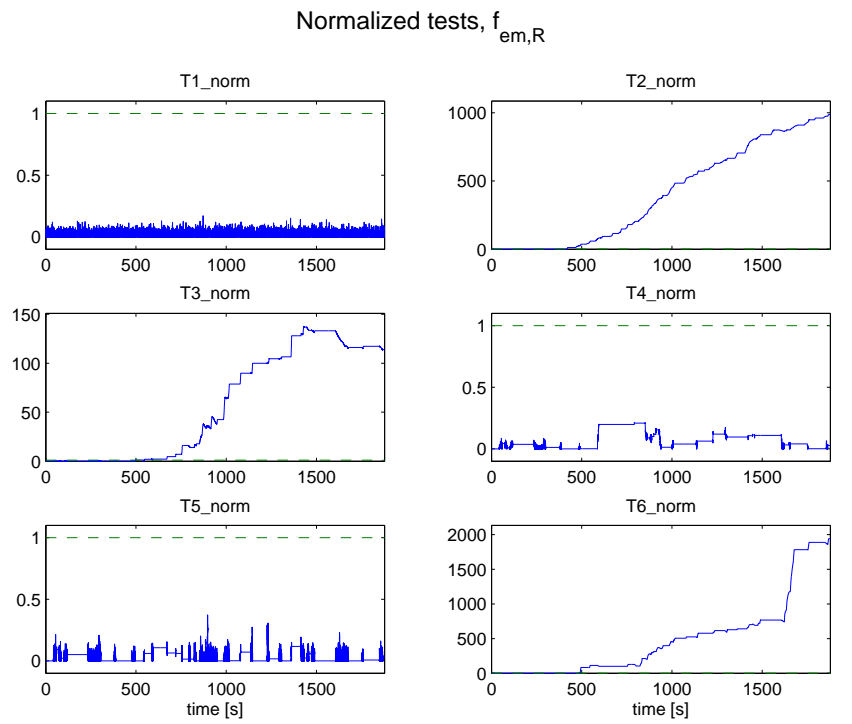

Fig. 5. The figure shows the normalized tests when there is a fault in the resistance in the electric machine. Test 4 does not react on the fault as it should do according to the structural analysis.

parameters in the CUSUM algorithm that changes with the operating points of the vehicle, to adapt to varying fault sensitivity.

For the five faults that are fully isolable the result is obtained within 100 seconds. There are a number of reasons that it takes longer than for Diagnosis system 1 . One reason is that an algebraic loop is not uniquely solved in one of the tests. Another reason is that some of the tests are not valid at all times, here because the model of the clutch is not valid in all operating modes, $\left|U_{e m}\right|$ is small, or that a low gear is used. In the four tests based on dynamic residual generators, the states in the filters need to be reinitialized when the system is reactivated. The assumption that the system is fault free is used in the reinitialization of the state, and an alternative to this, which possibly increases the performance of the diagnosis, is to instead use the previous valid value of the residual in the initialization.

\section{CONCLUSIONS}

In hybrid vehicles there are new features compared to conventional vehicles, like for example mode switches. The influence of these properties and of the sensor configuration on diagnosis has been studied by design, implementation and comparative simulation studies. Specifically, two diagnosis systems based on different sensor configurations are studied.

According to the structural analysis of the model, full fault isolability is possible in both sensor configurations. For the first sensor configuration, full isolability is achieved in the implemented diagnosis system. For the second case, full isolability is not reached by the implemented system, since for example, a change in the torque constant $k_{i}$ can not be isolated from a fault in the gearbox speed sensor. The discrepancy between the model analysis and the performance of the implemented diagnosis systems stems from the fact that the influence of the faults on the system in relation to the noise level is not taken into account in the model analysis.

There is no major complexity difference in the design and implementation between the two diagnosis systems. Due to the sensor configuration in the system based on few sensors, the tests are larger and therefore slightly more computational demanding.

The system model is not valid in all operating conditions, for example due to mode switching or the incomplete clutch model. This leads to that some tests are deactivated during a significant part of the time, which leads to decreased detection performance. When deactivations and activations of tests are common, fast and reliable initialization of the dynamic tests are important. Here, a well designed energy management procedure can choose a pattern of operating points, and thereby positively affect the diagnosis performance. In summary, the developed simulation platform has proven a suitable environment for studying overall monitoring and diagnosis for hybrid vehicle powertrains.

\section{REFERENCES}

Blanke, M., Kinnaert, M., Lunze, J., and Staroswiecki, M. (2006). Diagnosis and Fault-Tolerant Control. Springer, 2nd edition.

CAPSim (2009). http://www.capsim.se.

Chau, K., Chan, C., and Liu, C. (2008). Overview of permanent-magnet brushless drives for electric and hybrid electric vehicles. Industrial Electronics, IEEE Transactions on, 55(6), 2246 -2257. doi: 10.1109/TIE.2008.918403.

de Kleer, J., Mackworth, A., and Reiter, R. (1992). Characterizing diagnoses and systems. Artificial Intelligence, 56(2-3), 197-222.

Dustegör, D., Frisk, E., Coquempot, V., Krysander, M., and Staroswiecki, M. (2006). Structural analysis of fault isolability in the DAMADICS benchmark. Control Engineering Practice, 14(6), 597-608.

Frisk, E. and Nyberg, M. (2001). A minimal polynomial basis solution to residual generation for fault diagnosis in linear systems. Automatica, 37(9), 1417-1424.

Guzzella, L. and Sciarretta, A. (2007). Vehicle Populsion System, Introduction to Modelling and Optimization. Springer Verlag, Zürich, 2 edition.

Krysander, M. and Frisk, E. (2008). Sensor placement for fault diagnosis. IEEE Transactions on Systems, Man, and Cybernetics - Part A: Systems and Humans, 38(6), 1398-1410.

Lin, C.C., Peng, H., Grizzle, J., and Kang, J.M. (2003). Power management strategy for a parallel hybrid electric truck. Control Systems Technology, IEEE Transactions on, 11(6), 839 - 849. doi:10.1109/TCST.2003.815606.

Page, E. (1954). Continous inspection schemes. Biometrika, 41, 100-115.

Sciarretta, A. and Guzzella, L. (2007). Control of hybrid electric vehicles. IEEE CONTROL SYSTEMS MAGAZINE, 27(2), 60-70.

Sundström, C., Frisk, E., and Nielsen, L. (2010). A platform for overall monitoring and diagnosis for hybrid vehicle powertrains. Technical Report LiTH-R-2925, Department of Electrical Engineering, Linköpings Universitet, SE-581 83 Linköping, Sweden. 